\title{
NANOSCALE LIPOPHILIC PRODRUGS OF DEXAMETHASONE WITH ENHANCED \\ PHARMACOKINETICS
}

Mathilde Lorscheider ${ }^{1}$, Nicolas Tsapis ${ }^{1}$, Rosana Simón-Vázquez ${ }^{2}$, Nicolas Guiblin ${ }^{3}$, NourEddine Ghermani $^{1}$, Franceline Reynaud ${ }^{1,4}$, Romain Canioni ${ }^{1}$, Sonia Abreu ${ }^{5}$, Pierre Chaminade ${ }^{5}$, Elias Fattal ${ }^{1}$

${ }^{1}$ Institut Galien Paris-Sud, CNRS, Univ. Paris-Sud, Univ. Paris-Saclay, 92290 Châtenay-Malabry, France ${ }^{2}$ Immunology, Biomedical Research Center (CINBIO) and Institute of Biomedical Research of Orense, Pontevedra and Vigo (IBI), University of Vigo, Campus Lagoas Marcosende, Pontevedra, 36310, Spain ${ }^{3}$ École Centrale Paris, Laboratoire Structures, Propriétés et Modélisation des Solides (SPMS) UMR CNRS 8580, CentraleSupélec, Univ. Paris Saclay, 3 Rue Joliot Curie, 91190 Gif-sur-Yvette, France.

${ }^{4}$ School of Pharmacy, Federal University of Rio de Janeiro, 21944-59 Rio de Janeiro, Brazil.

${ }^{5}$ Lip(Sys) ${ }^{2}$ EA7357 Lipides, systèmes analytiques et biologiques, Univ. Paris-Sud, Univ. Paris-Saclay, 92290 Châtenay-Malabry, France

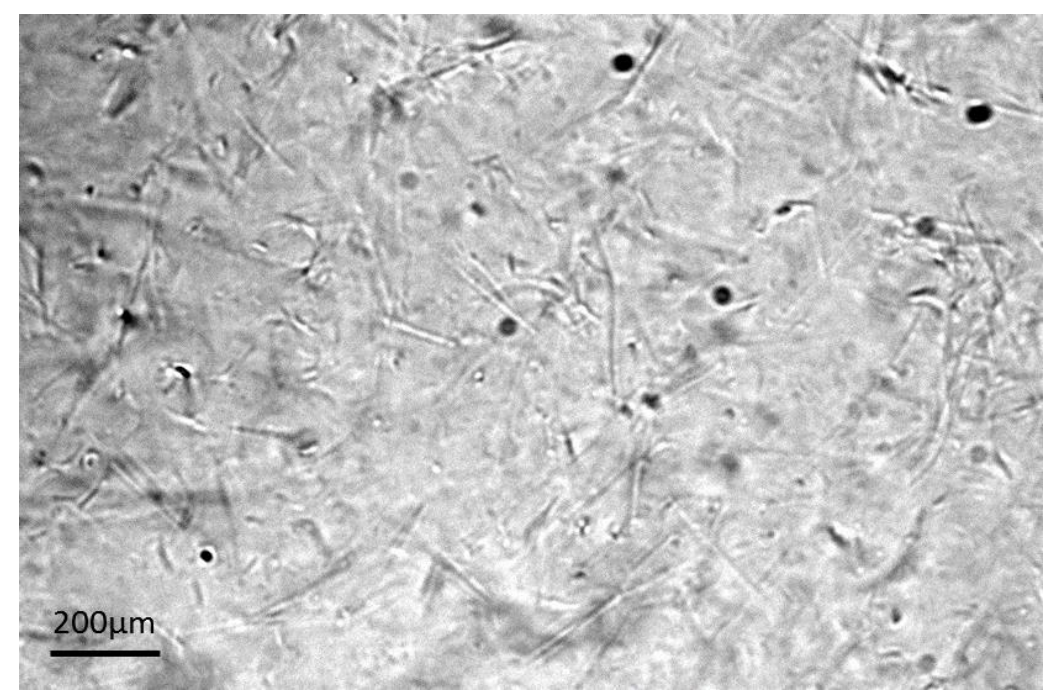

Figure S1. DXP crystals apparition after nanoparticles storage at room temperature during more than 37 days. 


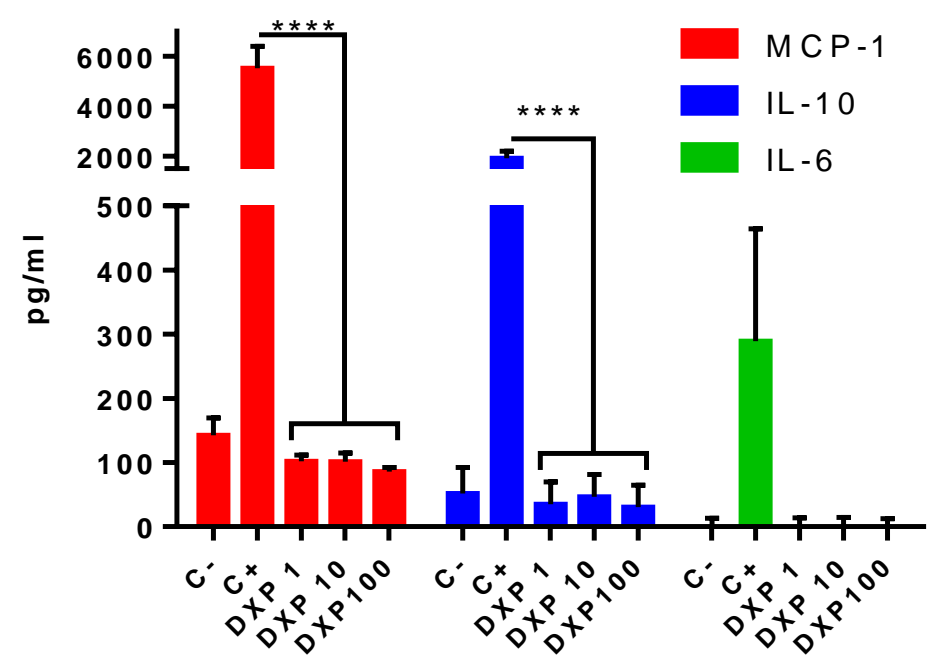

Figure S2. Cytokine production by Raw 264.7 macrophages with LPS induction (C+) or without LPS induction after exposure to medium (C-), DXP nanoparticles at $1 \mu \mathrm{g} / \mathrm{mLI}$ DXP (DXP1), 10 $\mu \mathrm{g} / \mathrm{mLI}$ (DXP10), 100 $\mathrm{g} / \mathrm{mLI}$ (DXP100). Three cytokines were evaluated: MCP-1 (red), IL-10 (blue) and IL-6 (green). $\mathrm{n}=3$ for all groups. Statistical analysis performed with two-way ANOVA followed by Tukey's post test. ${ }^{* * *} \mathrm{p}<0.0001$ indicates differences with positive control $(\mathrm{C}+)$. 